\title{
Corrosion d'origine microbienne sous les sols du karst du Nord-Est de la Hongrie. Étude expérimentale
}

Microbial corrosion under the karstic soils of North-East Hungary. Experimental study

Korrosion Mikrobischen Ursprungs unter den Karstböden im Nordöstlichen

Ungarn. Experimentelle Studie

\section{László Zámbó}

\section{OpenEdition}

Journals

Édition électronique

URL : http://journals.openedition.org/rge/3913

DOI : $10.4000 /$ rge.3913

ISSN : 2108-6478

Éditeur

Association des géographes de l'Est

Édition imprimée

Date de publication : 1 septembre 2001

ISSN : 0035-3213

Référence électronique

László Zámbó, «Corrosion d'origine microbienne sous les sols du karst du Nord-Est de la Hongrie. Étude expérimentale », Revue Géographique de l'Est [En ligne], vol. 41 / 4 | 2001, mis en ligne le 22 juillet 2013, consulté le 08 septembre 2020. URL : http://journals.openedition.org/rge/3913 ; DOI : https:// doi.org/10.4000/rge.3913

Ce document a été généré automatiquement le 8 septembre 2020

Tous droits réservés 


\title{
Corrosion d'origine microbienne sous les sols du karst du Nord-Est de la Hongrie. Étude expérimentale
}

Microbial corrosion under the karstic soils of North-East Hungary. Experimental study

Korrosion Mikrobischen Ursprungs unter den Karstböden im Nordöstlichen

Ungarn. Experimentelle Studie

\author{
László Zámbó
}

\section{Introduction}

1 D'après les recherches des dernières décennies, il est généralement admis que les processus de dissolution karstique sont générés par différents acides qui accèdent à la circulation de l'eau dans les karsts. Ces acides sont les agents principaux des réactions chimiques. Il est aussi admis que la source de ces acides se trouve dans le sol couvrant le karst, entretenant ainsi ce que l'on peut appeler l'« effet de sol sur la karstification ».

2 Toujours d'après la bibliographie spécialisée, dans la composition des acides provenant du sol, l'importance du CO2 est décisive pour les processus de dissolution. L'accord est général pour estimer que les acides qui contrôlent la dissolution des carbonates dans les sols, et en particulier l'acide carbonique, sont les produits de trois groupes de processus :

- la respiration par les racines des végétaux supérieurs,

- les processus de décomposition organique par les microorganismes ou microbiotes,

- d'autres processus d'altération chimique, d'origine non-microbienne.

Cependant, en ce qui concerne les parts respectives de ces processus dans le mécanisme de corrosion, il n'existe que des estimations sporadiques, indiquant notamment que ces trois processus sont sensiblement de même importance pour la production de C02. Le but de nos recherches est d'essayer de fournir une évaluation quantitative de ces 
phénomènes et de mesurer leurs parts respectives dans la corrosion karstique sous couvert pédologique.

\section{Méthodologie}

4 Pour obtenir des connaissances plus précises sur les processus de dissolution des carbonates sous les sols, on a choisi une méthode expérimentale. 96 petits appareils de simulation en grandeur réelle ont été construits à cet effet.

\section{A. L'appareillage}

Figure 1 : L’appareillage employé

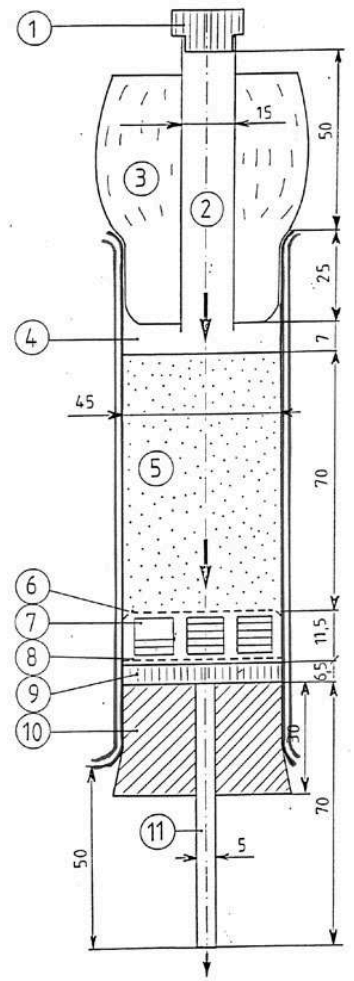

1,10 bouchons de caoutchouc ; 2 recharge de l'eau ; 3 filtre de bactéries ; 4 tube de verre ; 5 échantillon de sol ; 6,8,9 filtre plastique ; 7 cubes de calcaire de $1 \mathrm{~cm} 3$ de volume ; 11 solution infiltrée (dimensions en $\mathrm{mm}$ )

5 Il se compose d'un tube de verre de $40 \mathrm{~mm}$ de diamètre dans lequel on place un échantillon de sol d'une épaisseur de $9 \mathrm{~cm}$. Le tube est fermé par un bouchon filtrant les bactéries mais n'empêchant pas la circulation de l'air, et à travers lequel on verse de l'eau filtrée pour simuler les précipitations. Sous le sol, on place 7 cubes de $1 \mathrm{~cm} 3$ d'un calcaire bien soluble, entre deux filtres plastiques chimiquement inactifs, pour observer l'effet de la corrosion. La solution traverse donc successivement le sol, puis les cubes calcaires. Elle est recueillie dans un sac plastique stérile à la base de l'appareil.

6 La capacité de dissolution du calcaire est exprimée par la concentration en carbonates dissous (en milligrammes) trouvée dans la solution recueillie, ou par la quantité équivalente de $\mathrm{CO} 2$. 


\section{B. Les conditions expérimentales}

7 Les échantillons ont été recueillis dans le karst d'Aggtelek, dans la région nordorientale de la Hongrie.

8 Le fonctionnement des appareils a été étudié en se plaçant dans les conditions climatiques moyennes de la région: neuf mois sans gel, $600 \mathrm{~mm}$ de précipitations annuelles.

9 Quatre types de sols karstiques parmi les plus caractéristiques de la zone tempérée et subtropicale ont été choisis :

- une rendzine noire avec 95 à $98 \%$ de matière organique et 2 à $5 \%$ d'argile,

- une rendzine brune avec 50 à $80 \%$ de matière organique, 20 à $52 \%$ de débris calcaires, un peu d'argile,

- une terre humifère brun-rouge avec 2 à $5 \%$ de matière organique, 95 à 98 \% d'argile,

- une terra rossa relictuelle avec 0,5 à $2 \%$ de matière organique, 98 à 99,5\% d'argile.

Chaque type de sol est soumis aux expérimentations suivantes:

- dans 4 appareils, on place le sol originel avec ses microorganismes, de manière à mesurer la capacité de dissolution naturelle ;

- dans 4 autres appareils, on place un échantillon de sol préalablement stérilisé, de manière à mesurer la capacité de dissolution en dehors de l'action microbienne ;

- dans 8 autres appareils à échantillon de sol stérile, on ré-inocule séparément les 8 types de bactéries les plus communes ;

- dans 8 autres appareils à échantillon de sol stérile, on ré-inocule séparément 8 types de Streptomycetes, de manière à étudier l'effet spécifique de ces microorganismes sur la capacité de dissolution des carbonates. Ces 8 espèces les plus communes de bactéries et de Streptomycetes avaient été choisies parmi 160 espèces déterminées lors de recherches précédentes.

11 L'effet de la corrosion est mesuré par trois méthodes, de manière à pouvoir comparer les résultats :

- analyse de l'eau infiltrée : mesure de la quantité totale de carbonates, de la quantité de $\mathrm{CaCO} 3$ et de $\mathrm{MgCO} 3$, en milligrammes ;

- perte de poids des cubes de calcaire, en milligrammes ;

- mesure de la rugosité de la surface des cubes de calcaire par microscopie couplée à un ordinateur.

\section{Discussion sur les modalités d'expérimentation}

\section{Epaisseur de l'échantillon et horizon pédologique concerné}

Nos études précédentes (Zámbó, 1986, 1992 ; Zámbó, Darabos, 1993) avaient montré que l'épaisseur des sols joue un rôle important dans la capacité de dissolution des carbonates. En étudiant l'activité microbienne des sols, Bárány-Kevei (1992) a fait la même constatation. D'après White (1988), la production de CO2 est maximale dans les horizons 0 et $\mathrm{A}$ des sols, c'est-à-dire à leur partie supérieure. Mais inversement, Crowther $(1983,1984)$ a mesuré le contenu moyen de CO2 dans les sols et a constaté que la concentration totale de $\mathrm{CO} 2$ augmente vers le bas. En effet, si la quantité de matière organique, le nombre et l'activité des microbes diminuent vers le bas, donc si la 
production biogénique de $\mathrm{CO} 2$ augmente vers la surface, la perte en $\mathrm{CO} 2$ qui se diffuse dans l'air s'élève aussi avec la porosité et affaiblit cette teneur. En conséquence, la circulation de l'air au sein du sol est un facteur déterminant pour l'explication de la variation de la concentration en $\mathrm{CO} 2$.

La concentration réelle en $\mathrm{CO} 2$ dans le sol dépend donc de l'équilibre entre le $\mathrm{CO} 2$ dissous dans l'eau infiltrée et celui qui se trouve dans l'air du sol. Selon les expériences de Reardon, Allison et Fritz (1979), la production et le transport de CO2 sont surtout effectués dans la zone des racines et en-dessous de celle-ci, cette zone étant considérée comme la partie non-saturée du système eau-gaz. La plus grande partie du $\mathrm{CO} 2$ produit dans le sol se diffuse dans l'air: la perte en $\mathrm{CO} 2$ est donc fonction des propriétés physiques des sols. Ainsi, la diffusion du gaz est plus facile au sein des sols meubles, mais les sols argileux conservent mieux le CO2 (White, 1988). Ces différences de comportement des différents types de sols (humifères ou argileux) apparaissent nettement dans nos résultats.

Pour avoir des résultats comparables entre eux, nous avons donc prélevé les échantillons toujours de la même manière, à la partie supérieure des sols, qui est la plus riche en humus. Nos résultats se rapportent donc aux horizons pédologiques les plus riches en $\mathrm{CO} 2$ produit au sein du sol et susceptible de se diffuser plus ou moins en fonction des propriétés physiques du sol considéré.

Dans nos expérimentations, le système de filtration est un système ouvert dans lequel les concentrations en $\mathrm{CO} 2$ dissous dans l'eau infiltrée et en $\mathrm{CO} 2$ gazeux du sol sont en équilibre. La concentration supplémentaire en $\mathrm{CO} 2$ qui va provoquer la corrosion des cubes de calcaire est donc bien le résultat de l'action des microorganismes décomposant la matière organique du sol.

\section{Conditions de température et humidité}

16 On sait que la production de $\mathrm{CO} 2$ dans le sol dépend des conditions d'humidité et de température (Gerstenhauer, 1972; White, 1988). Miotke (1974) et Atkinson (1977) ont déterminé une relation directe entre la température du sol et la concentration en CO2. Anderson (1973), Garett et Cox (1973), Edwards (1975), Jakucs (1977) ont recherché la relation entre l'humidité du sol et la production de CO2 ; enfin Gunn et Trudgill (1982) ont établi que température et humidité du sol sont des facteurs déterminants de la production de $\mathrm{CO} 2$ durant la période végétative, mais que la relation entre la concentration en $\mathrm{CO} 2$ et les conditions météorologiques ne pouvait se résoudre à une équation simple. De même, on peut lire dans Gewelt et Ek (1986) : "la relation entre l'évolution des teneurs en $\mathrm{CO} 2$ et la source de $\mathrm{CO} 2$ (le sol) est nette au niveau des variations annuelles qui paraissent bien refléter le rythme saisonnier de la biomasse, avec son minimum d'activité en hiver ».

17 Ainsi, pendant la durée des mesures considérées (du 16/12/95 au 12/06/96), sur l'ensemble des échantillons, les températures ont varié de manière identique entre 7 et $20^{\circ}$, excluant le gel (Fig. 2a). La recharge en eau étant effectuée en même temps, les variations d'humidité ont également été identiques pour tous les échantillons (Fig. 2b), les valeurs réelles de l'humidité étant bien sûr différentes d'un échantillon à l'autre. 
Figure 2a : Variations de la température au cours de l'expérimentation

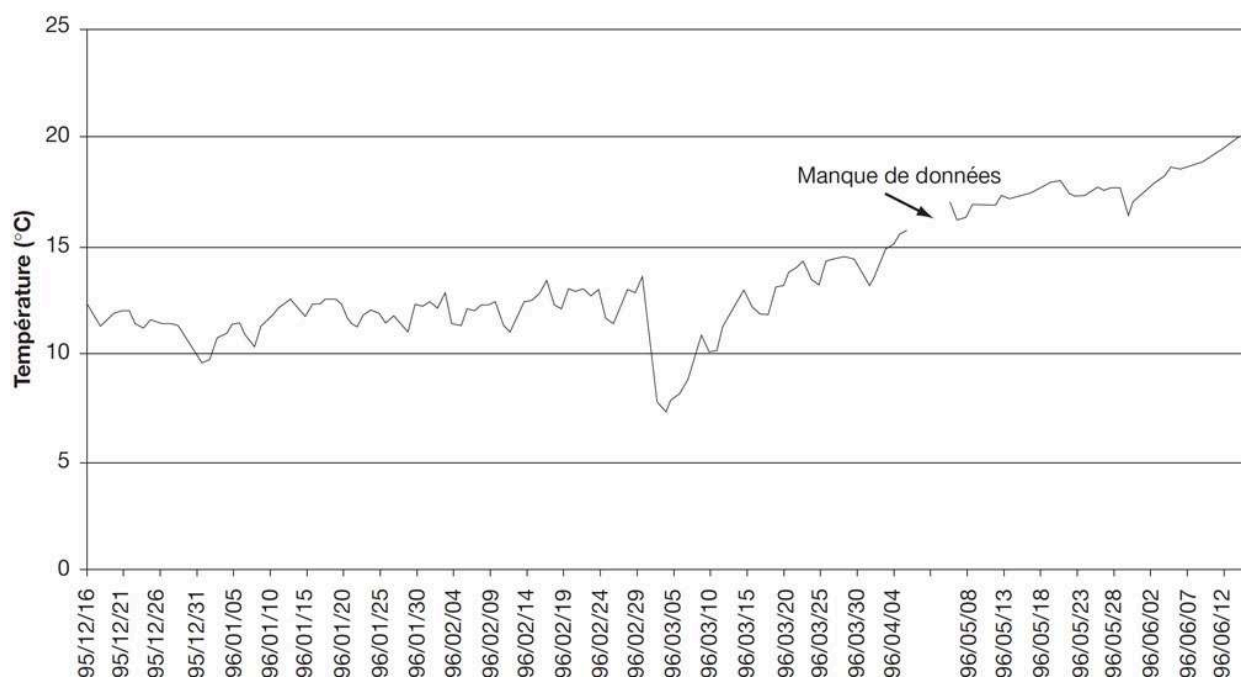

Figure $2 b$ : Variations de l'humidité au cours de l'expérimentation

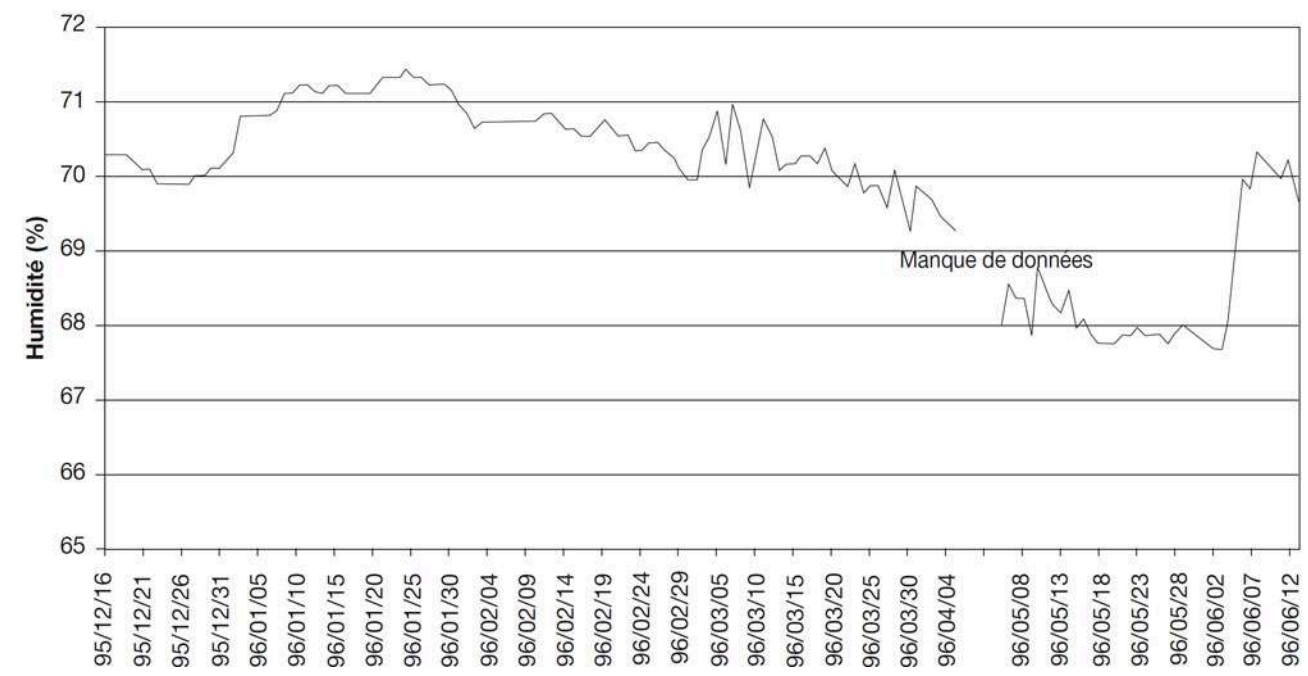

\section{Principaux résultats}

\section{A. Résultats généraux : la corrosion microbienne}

Le Tableau 1 montre que dans tous les cas, la concentration en carbonates dans la solution infiltrée, c'est-à-dire la dissolution des carbonates qui mesure la corrosion, est toujours plus importante en présence des microorganismes (les moyennes des séries «stériles » sont toujours les plus faibles). Les expériences confirment donc d'abord que l'action microbienne est primordiale. Les moyennes des séries B (action de 8 bactéries) et $S$ (action de 8 Streptomycetes) sont à peu près identiques; l'action conjuguée de l'ensemble des microorganismes n'est cependant guère plus élevée que l'effet d'un seul type de bactérie ou de Streptomycète. Cela signifie que la diversité des microorganismes n'influence pas tellement la capacité de corrosion. Une application très importante de cette observation est qu'une modification accidentelle du milieu par 
une pollution détruisant certaines bactéries n'entraîne pas nécessairement une baisse de l'intensité de la corrosion.

Tableau 1 : Concentration en carbonates dans la solution infiltrée (en $\mathrm{mg} / \mathrm{l})$

\begin{tabular}{|c|c|c|c|c|}
\hline Contenu du tube & Rendzine noire & Rendzine brune & Terre brun-rouge & Terra rossa \\
\hline $\begin{array}{l}\text { Originel } 1 \\
\text { Originel } 2 \\
\text { Originel } 3 \\
\text { Originel } 4\end{array}$ & $\begin{array}{r}637,98 \\
655,00 \\
1096,59\end{array}$ & $\begin{array}{r}470,02 \\
1218,46\end{array}$ & $\begin{array}{l}185,98 \\
210,01 \\
156,37\end{array}$ & $\begin{array}{r}80,00 \\
121,98 \\
41,62\end{array}$ \\
\hline Moyenne origine & 796,52 & 844,24 & 184,12 & 81,20 \\
\hline $\begin{array}{l}\text { B1 } \\
\text { B2 } \\
\text { B3 } \\
\text { B4 } \\
\text { B5 } \\
\text { B7 } \\
\text { B8 } \\
\text { B10 }\end{array}$ & $\begin{array}{r}610,00 \\
566,09 \\
455,01 \\
549,00 \\
569,99 \\
518,01 \\
492,04 \\
1000,00\end{array}$ & $\begin{array}{r}368,46 \\
240,02 \\
281,72 \\
329,99 \\
374,98 \\
0,00 \\
57,11 \\
370,01 \\
\end{array}$ & $\begin{array}{l}140,00 \\
129,29 \\
170,00 \\
271,99 \\
104,99 \\
211,99 \\
205,96 \\
176,01\end{array}$ & $\begin{array}{r}74,99 \\
224,99 \\
69,00 \\
76,99 \\
151,97 \\
81,99 \\
308,99 \\
98,31 \\
\end{array}$ \\
\hline Moyenne sér. B & 595,02 & 252,79 & 176,28 & 135,90 \\
\hline $\begin{array}{l}\text { S1 } \\
\text { S2 } \\
\text { S3 } \\
\text { S4 } \\
\text { S5 } \\
\text { S7 } \\
\text { S8 } \\
\text { S10 }\end{array}$ & $\begin{array}{l}473,00 \\
689,98 \\
400,00 \\
534,98 \\
454,99 \\
500,00 \\
291,00 \\
439,00 \\
\end{array}$ & $\begin{array}{l}375,89 \\
287,45 \\
335,00 \\
299,00 \\
320,00 \\
195,01 \\
224,99 \\
334,98 \\
\end{array}$ & $\begin{array}{l}100,00 \\
128,00 \\
148,98 \\
200,08 \\
171,99 \\
100,55 \\
178,00 \\
104,99 \\
\end{array}$ & $\begin{array}{r}80,01 \\
93,00 \\
52,00 \\
36,02 \\
89,99 \\
236,04 \\
81,99 \\
126,03 \\
\end{array}$ \\
\hline Moyenne sér. S & 472,87 & 296,54 & 141,57 & 99,38 \\
\hline Moyenne B et S & 533,94 & 274,66 & 158,93 & 117,64 \\
\hline $\begin{array}{l}\text { Stérile } 1 \\
\text { Stérile } 2 \\
\text { Stérile } 3 \\
\text { Stérile } 4\end{array}$ & $\begin{array}{l}142,14 \\
101,54\end{array}$ & $\begin{array}{r}114,78 \\
73,17\end{array}$ & $\begin{array}{l}30,99 \\
38,61 \\
39,10\end{array}$ & $\begin{array}{c}47,74 \\
30,97 \\
21,31\end{array}$ \\
\hline Moyenne stérile & 121,84 & 93,97 & 36,24 & 33,34 \\
\hline
\end{tabular}

$\mathrm{B} 1$ à $\mathrm{B} 10$ : bactéries ré-inoculées ; S1 à S10 : streptomycètes ré-inoculés 
Tableau 2 : Carbonate de magnésium dissous (en $\mathrm{mg}$ )

\begin{tabular}{|l|c|c|c|c|}
\hline \multicolumn{1}{|c|}{ Contenu du tube } & Rendzine noire & Rendzine brune & Terre brun-rouge & Terra rossa \\
\hline Originel 1 & 20,96 & 20,70 & 10,47 & 10,26 \\
Originel 2 & 15,91 & & 15,43 & 12,38 \\
Originel 3 & 39,07 & 29,70 & & \\
Originel 4 & & & 30,44 & 14,26 \\
\hline Moyenne origine & 25,31 & 25,20 & 18,78 & 12,30 \\
\hline B1 & 39,71 & 30,40 & 19,34 & 7,41 \\
B2 & 68,78 & 20,04 & 21,27 & 37,32 \\
B3 & 58,31 & 19,38 & 25,08 & 12,61 \\
B4 & 34,64 & 17,91 & 39,80 & 13,18 \\
B5 & 31,83 & 37,59 & 19,09 & 18,20 \\
B7 & 22,70 & & 30,17 & 8,95 \\
B8 & 41,24 & 14,26 & 17,82 & 32,86 \\
B10 & 36,10 & 53,78 & 28,91 & 15,03 \\
\hline Moyenne sér. B & 41,66 & 27,62 & 25,19 & 18,20 \\
\hline S1 & 37,47 & 24,71 & 8,86 & 12,60 \\
S2 & 9,37 & 33,41 & 17,44 & 5,18 \\
S3 & 24,76 & 46,52 & 17,84 & 4,27 \\
S4 & 22,30 & 25,48 & 7,80 & 4,82 \\
S5 & 21,50 & 40,64 & 31,22 & 10,00 \\
S7 & 31,06 & 21,88 & 13,37 & 26,93 \\
S8 & 23,70 & 9,27 & 24,06 & 6,55 \\
S10 & 37,80 & 24,14 & 4,28 & 4,99 \\
\hline Moyenne sér. S & 26,00 & 28,26 & 15,61 & 9,42 \\
\hline Moyenne B et S & 33,83 & 27,94 & 20,40 & 13,81 \\
\hline Stérile 1 & & & & \\
Stérile 2 & 7,72 & 12,94 & 5,82 & 5,52 \\
\hline Stérile 3 & & & 7,04 & \\
Stérile 4 & 6,53 & 9,11 & 6,57 & \\
\hline Moyenne stérile & 7,13 & & & \\
\hline & & & & \\
\hline & & & & \\
\hline
\end{tabular}

B1 à B10 : bactéries ré-inoculées ; S1 à S10 : streptomycètes ré-inoculés

\section{B. Influence du type de sol sur la corrosion}

\section{Type de sol et dissolution totale}

19 La capacité de dissolution mesurée par la quantité totale de carbonates dissous varie selon les types de sols. Sous les rendzines, la capacité de dissolution d'origine microbienne naturelle (Tableau 1, moyennes « orig.») est de 6,5 à 9 fois supérieure à celle des dispositifs stériles. Sous les sols argileux, cette proportion tombe à 5 , voire à 2,5. La corrosion globale serait donc plus importante au sein des rendzines qu'au sein des sols argileux.

\section{Type de sol et mécanismes de corrosion}

Le Tableau 1 montre que dans tous les cas, la concentration en carbonates dans l'eau infiltrée est nettement plus forte sous les rendzines que sous les sols argileux. Pourtant, la quantité d'eau recueillie sous les rendzines est plus faible (170 à $350 \mathrm{ml} / \mathrm{mn}$ ) que sous les sols argileux (250 à $420 \mathrm{ml} / \mathrm{mn}$ ). Dans les rendzines à structure aérée, les échanges gazeux, la diffusion du $\mathrm{CO} 2$ et l'évaporation sont plus intenses, ce qui explique cette contradiction apparente. Grossièrement, on peut établir que dans le cas des échantillons originels, la quantité totale de carbonates dissous est proportionnelle au contenu du sol en matières organiques.

21 Mais les observations de la diminution de la masse des cubes de calcaire (comme d'ailleurs les observations in situ) montrent que sous les rendzines, la karstification de la roche saine est beaucoup moins intense que sous les sols argileux : la corrosion des 
cubes de calcaire est plus forte sous les sols argileux que sous les rendzines (Fig. 3). C'est donc que la capacité de dissolution pourtant nettement supérieure au sein des rendzines est utilisée durant l'infiltration à dissoudre les débris calcaires encore présents dans ce type de sol, ce qui n'est pas le cas dans les sols argileux, où l'ensemble des acides produits dans le sol contribue à la corrosion des calcaires sous-jacents. D'ailleurs les sols argileux peuvent même subir une recalcification par remontée capillaire et reprécipitation des carbonates dissous dans l'eau qui avait atteint le substratum (Fig. 4). Cette observation fournit sans doute une explication à la transformation de sols argileux en rendzines.

Figure 3 : La perte de poids des cubes de pierre calcaire

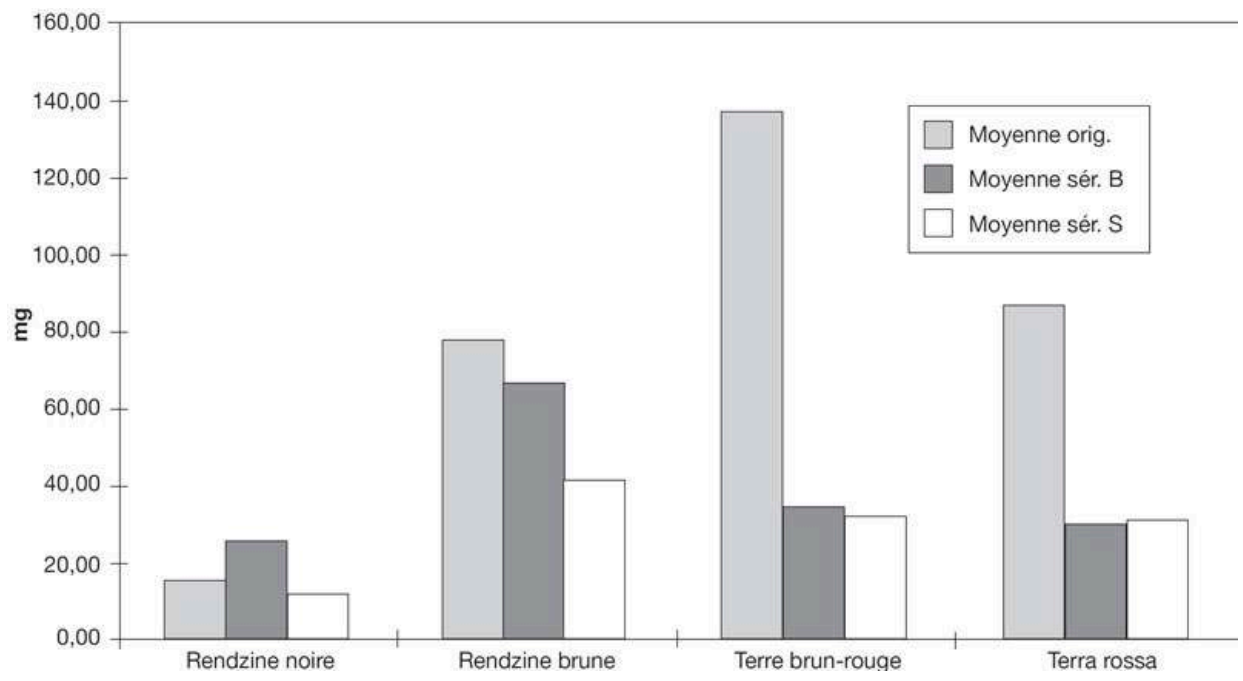

Figure 4 : Le changement de contenu en carbonates dans les sols

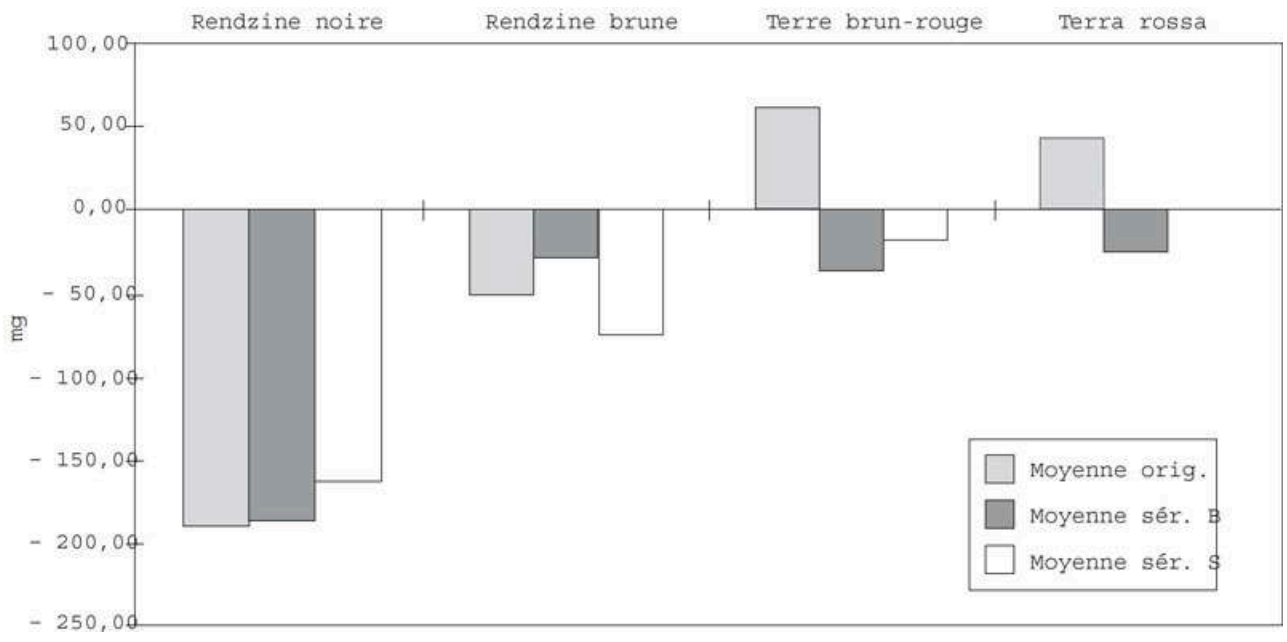

\section{Dissolution et nature des carbonates}

Les teneurs en carbonate de magnésium dissous (Tableau 2) sont toujours beaucoup plus faibles que celles en carbonates de calcium. La différence de comportement entre les rendzines et les sols argileux y est cependant beaucoup moins marquée. Comme de plus, on voit que pour le carbonate de magnésium la part des processus non-microbiens 
est proportionnellement beaucoup plus forte que pour le carbonate de calcium, cette moindre dépendance vis-à-vis de la dissolution microbienne s'explique.

Pour la dissolution d'origine microbienne, on peut cependant remarquer quelques différences de comportement selon les types de sols : pour les rendzines noires, l'effet de certaines souches de bactéries (B2 et B3) est plus important; pour les rendzines brunes, ce sont les Streptomycetes S3 et S5 qui se montrent les plus actifs. Des investigations complémentaires seraient cependant ici souhaitables avant de conclure à la nécessité de la présence de telle ou telle espèce pour la dissolution en milieu dolomitique.

\section{Conclusion}

Le résultat le plus important de ces expérimentations est que la corrosion des calcaires sous les sols dépend essentiellement du type de sol. Les expériences ont montré que les processus de dissolution varient selon les différents types de sols: la capacité de dissolution est déterminée par le contenu et les apports éventuels en matière organique. Le processus dépend aussi de l'épaisseur du sol et surtout de sa structure qui détermine la circulation de l'air et donc la diffusion du $\mathrm{CO} 2$ ainsi que l'évaporation. Si le potentiel de dissolution des rendzines, sols aérés, est supérieur à celui des sols argileux compacts, on constate cependant que la corrosion est plus active sous ces derniers, d'abord parce qu'il y parvient plus d'eau et parce que cette eau est encore active (dans les rendzines aérées, elle s'évapore en partie et elle dissout les particules carbonatées encore présentes dans le sol).

Le second résultat majeur est que dans tous les cas, le processus de dissolution d'origine microbienne est en moyenne de l'ordre de 4 fois plus important que les autres mécanismes.

Nous envisageons à la fois maintenant des recherches complémentaires et des adaptations du modèle expérimental à d'autres conditions climatiques pour affiner nos méthodes et conclusions.

\section{BIBLIOGRAPHIE}

Anderson J.M. (1973). - Carbon dioxide evolution from two temperate, deciduous woodland soils. Journal of Applied Ecology, 10, p. 361-378.

Atkinson T.C. (1977). - Carbon dioxide in atmosphere of the unsaturated zone : an important control of groundwater hardness in limestones. Journal of Hydrology, 35, p. 111-123.

Bárány-Kevei I. (1992). - Ecological regulation of karst development. New perspectives in Hungarian Geography. Akadémiai Kiadó, p. 77-80.

Crowther J.L. (1983). - Carbon dioxide concentrations in some tropical karst soils, West-

Malaysia. Catena, 10, p. 27-39. 
Crowther J.L. (1984). - Soil carbon dioxide and weathering potentials in tropical karst terrain, Peninsular Malaysia : a preliminary model. Earth Surface Processes and Land-forms, 9, p. 397-407.

Edwards N.T. (1975). - Effect of temperature and moisture on carbon-dioxide evolution in a mixed deciduous forest floor. Soil Science Society of America, Proceedings 37, p. 361-365.

Garrett H.E. and Cox G.S. (1973). - Carbon dioxide evolution from the floor of an Oak-Hickory forest. Soil Science Society of America, Proceedings 37, p. 641-644.

Gerstenhauer A. (1972), - Der Einfluss des CO2-gehaltes der Bodenluft auf die Kalklösung. Erdkunde, 26, p. 116-120.

Gewelt M. et Ek C. (1986). - L'évolution saisonnière de la teneur en CO2 de l'air de deux grottes belges : Sainte-Anne et Brialmont, à Tilff, p. 49-76. In : Paterson K. et Sweeting M.M. (ed.) : New Directions in Karst, Norwich, England, 613 p.

Gunn J. and Trudgill S.T. (1982). - Carbon dioxide production and concentrations in the soil atmosphere : a case study from New-Zealand vulcanic ash soils. Catena, 9, p. 81-94.

Jakucs L. (1977). - Morphogenetics of Karst Regions. Aka-démia Kiadó, Budapest.

Miotke F.-D. (1974). - Die Messung des CO2-gehaltes der Bodenluft mit dem Dräger-Gerät und die beschleunigte Kalklösung durch höhere Fliessgeschwindigereiten. Zeit-schrift für Geomorphologie, 16(1), p. 93-102.

Reardon E.I., Allison G.B. and Fritz P. (1979). - Seasonal chemical and isotopic variations of soil $\mathrm{CO} 2$ at Trout Creek, Ontario. Journal of Hydrology, 43, p. 355-371.

White W.B. (1988). - Geomorphology and Hydrology of Karsts Terrains, New York, Oxford Univ. Press, 464 p.

Zámbo L. (1986). - Characteristic values of karst-corrosion soil impact in the soil cover of dolines. Annales Univ. Sci. Budapestinensis de R. Eötvös Nominatae, Sectio Geogra-phia. Tom. XX-XXI, p. 311-325.

Zámbó L. (1992). - The soil effect in karst corrosion. New Perspectives in Hungarian Geography, Akadémiai Kiadó, Budapest, p. 81-90.

Zámbó L., Darabos G. (1993). - An investigation of microbial CO2 production in karst soils. In : Zámbó L. (ed.), Conference on the Karst and Cave Research in Hungary, Jósvafõ, p. 141-146.

\section{RÉSUMÉS}

Les acides qui contrôlent la dissolution des carbonates sous les sols sont les produits de la respiration par les racines des végétaux supérieurs, des processus de décomposition organique par les microorganismes, et d'autres processus d'altération chimique non microbienne. Pour une connaissance plus précise de la part respective de ces phénomènes, des expériences de corrosion en milieu contrôlé ont été réalisées. 96 exemplaires d'un montage expérimental ont été construits pour mesurer la corrosion sous les sols. Les 4 types les plus caractéristiques de sols karstiques de la zone tempérée et subtropicale (différentes rendzines et terres rouges argileuses) ont été placés dans les appareils. Pour chaque type de sol, 4 appareils contenaient des échantillons à microorganismes originels pour mesurer la capacité de dissolution naturelle tandis que 4 autres appareils à échantillons stérilisés mesuraient la capacité de dissolution nonmicrobienne. 16 autres appareils étaient dévolus à l'étude de l'action de bactéries spécifiques. La capacité de dissolution (= quantité totale de carbonates dissous) montre des valeurs différentes 
selon les types de sols. Pour l'ensemble des sols, la dissolution d'origine microbienne est beaucoup plus importante que celle des autres processus, mais ses proportions diminuent des rendzines aux sols argileux, ce qui montre l'importance de la matière organique. Pourtant, la corrosion est plus forte sous les sols argileux, où toute la capacité de dissolution est utilisée, alors que dans les rendzines une part de cette capacité s'évapore (sols aérés) ou est utilisée à dissoudre les carbonates encore présents dans le sol.

Acids that control the dissolution of carbonates under soils are products of respiration by overlying vegetation, of processes of organic decomposition by micro-organisms, and other chemical, non-microbial, alteration processes. For a more precise understanding of the respective contribution of these phenomena some controlled corrosion experiments had to be carried out. 96 experimental assemblages were constructed in order to measure subsoil corrosion. The 4 types most characteristic of karstic soils of the temperate and subtropical zone (different rendzinas and clayey terra rossas) were placed in the apparatus. For each type of soil, 4 pieces of apparatus containing samples with original micro-organisms would measure the capacity of natural dissolution, while 4 other pieces of apparatus with sterilised samples would measure the capacity of non-microbial dissolution. 16 other pieces of apparatus were employed to study the action of specific bacteria. The capacity of dissolution (= total quantity of dissolved carbonates) showed different values for each type of soil. For the entire suite of soils, microbial dissolution is much more important than the other processes but their proportions diminish from rendzinas to clayey soils, which shows the importance of the organic matter. Yet, corrosion is stronger under clayey soils, where all the dissolution capacity is utilised, whereas in the rendzinas part of this capacity evaporates (well-ventilated soils) or is used to dissolve carbonates still present in the soil.

Die Säuren, welche die Lösung der Karbonate unter den Böden bestimmen, sind das Ergebnis der Atmung durch die Wurzeln der Oberflächenvegetation, der Prozesse organischer Zersetzung durch die Mikroorganismen und anderer, nicht mikrobischer Prozesse chemischer Veränderung. Für eine genaue Kenntnis des jeweiligen Anteils dieser Phänomene wurden kontrollierte Korrosionsversuche durchgeführt. 96 Fälle einer Versuchsmontage wurden angelegt, um die Korrosion unter den Böden zu messen. Die vier am meisten charakteristischen Typen von Karstböden der gemässigten und subtropischen Zone (verschiedene Rendzinen und tonige Roterden) wurden in die Apparate gelegt. Für jeden Bodentyp enthielten vier Apparate Proben organischer Mikroorganismen, um die natürliche Lösungskapazität zu messen, während vier andere Apparate mit sterilisierten Proben die nicht mikrobische Lösungskapazität massen. 16 andere Apparate waren dem Studium spezifischer Bakterien-aktion gewidmet. Die Lösungskapazität (Gesamtmenge gelöster Karbonate) zeigt verschiedene Werte je nach Bodentypen. Für die Gesamtheit der Böden ist die Lösung mikrobischen Ursprungs viel wichtiger als die anderer Prozesse, aber die Anteile vermindern sich von den Rendzinen zu den tonigen Böden. Dies zeigt die Bedeutung der organischen Materie. Dennoch ist die Korrosion unter den Tonböden stärker, wo die ganze Lösungskapazität genutzt wird, während in den Rendzinen ein Teil dieser Kapazität verdunstet (« sols aérés ») oder genutzt wird, um die noch im Boden vorhandenen Karbonate zu lösen. 
INDEX

Schlüsselwörter : Experimentation, Karst, Korrosion, Lösung, Mikroorganismen, Rendzinen, Terra rossa, Ungarn

Keywords : corrosion, dissolution, experimentation, Hungary, karst, microorganisms, rendzina, terra rossa

Mots-clés : corrosion, dissolution, expérimentation, Hongrie, karst, microorganismes, rendzine, terra rossa

\section{AUTEUR}

\section{LÁSZLÓ ZÁMBÓ}

Université Eötvös Loránd, Département de Géographie physique, 2, Ludovika tér, 1083 Budapest, Hongrie 